\title{
Comorbilidad neuropsiquiátrica en pacientes con parálisis cerebral. Estudio longitudinal de cohorte
}

\section{Comorbidities in patients with cerebral palsy. Longitudinal cohort study}

\author{
Adalberto González-Astiazarán, ${ }^{*}$ Miguel Ángel Collado-Corona, ${ }^{*}$ Javier Terrazo-Lluch, ${ }^{*}$ \\ Paul Shkurovich-Bialik, ${ }^{*}$ Ernesto Ramírez-Navarrete, ${ }^{*}$ Emilio Arch-Tirado, ${ }^{*}$ Enrique De Font-Réaulx ${ }^{*}$
}

Citar como: González-Astiazarán A, Collado-Corona MÁ, Terrazo-Lluch J, Shkurovich-Bialik P, Ramírez-Navarrete E, Arch-Tirado E et al. Comorbilidad neuropsiquiátrica en pacientes con parálisis cerebral. Estudio longitudinal de cohorte. An Med (Mex). 2020; 65 (3): 176-181. https://dx.doi.org/10.35366/95673

\section{RESUMEN}

Introducción: La parálisis cerebral infantil es una alteración neurológica crónica con diferentes manifestaciones, repercusiones y terapéutica. La prevalencia global de 1.2$3.6 / 1,000$ nacidos vivos, cifra estable en las últimas tres décadas en diferentes países, independientemente de variables socioeconómicas. La prematurez y peso bajo al nacer son condiciones con mayor riesgo relativo para parálisis cerebral infantil. La comorbilidad es heterogénea y con frecuencia tiene igual o mayor importancia que la condición original. Objetivo: Definir las características de las condiciones comórbidas en una población con parálisis cerebral en seguimiento regular. Material y métodos: Estudio longitudinal de cohorte con diagnóstico y clasificación de parálisis cerebral infantil, y las comorbilidades neuropsiquiátricas. Se evaluaron pacientes con parálisis cerebral infantil, en seguimiento regular desde enero de 2013 hasta diciembre de 2019. La comorbilidad neuropsiquiátrica se definió como la coexistencia de alteraciones neurológicas o de enero de 2013 a diciembre de 2019. La distribución por sexo presenta predominio de neuropsiquiátrica. Resultados: Se evaluaron 287 pacientes con parálisis cerebral infantil, se distribuyeron por grupos de edad desde un mes hasta 57 años, media de nueve años ocho meses. La encefalopatía hipóxica es la primera causa, siendo 212 pacientes (73.86\%). Setenta pacientes con parálisis

\section{ABSTRACT}

Introduction: Cerebral Palsy is a chronic neurologic malady with different degrees of symptomatology, repercussion, and therapeutic schemes. The global prevalence of Cerebral Palsy is 1.2-3.6/1,000 live newborns, this figure has been stable in the last three decades in different countries, with few differences related to socioeconomic variables. Prematurity and low birth weight remain as the most important ones. The type of comorbidity is heterogeneous, frequently has the same or more importance for the medical management. Objective: To define the characteristics of the comorbidity in a population with cerebral palsy in regular clinical follow up. Material and methods: Is a longitudinal cohort study, in which we established diagnosis and classification of the primary condition and the presence of neuropsychiatric comorbidity we included patients on regular follow up from January 2013 to December 2019. Neuro psychiatric comorbidity was defined as the coexistence of abnormal conditions either primary neurologic or psychiatric conditions. Results: This series has 287 patients with Cerebral Palsy, on regular follow up from January 2013 to December 2019. The sex distribution has subtle predominance for male patients. Age distribution from one month to 57 years. The median age is nine years eight months. Hypoxic encephalopathy was the etiology in 212 cases (73.86\%). Seventy patients have no comorbidity (24.39\%), 84 patients (29.26\%) have a single
* Clínica de Epilepsia del Centro Neurológico del Centro Médico ABC.

Recibido para publicación: 30/05/2020. Aceptado: 10/08/2020.

\section{Alcaldía Miguel Hidalgo, CDMX.}

Tels. 555540 1730/55 52824183.

E-mail: neuropediatriayaprendizaje@gmail.com
Correspondencia:

Adalberto González-Astiazarán

Diego de Osorio Núm. 140 P.B.,

Col. Lomas de Chapultepec, 11000,

\author{
Abreviaturas: \\ $\mathrm{PCl}=$ Parálisis cerebral infantil. \\ $\mathrm{CNP}=$ Comorbilidades neuropsiquiátricas . \\ SNC = Sistema nervioso central. \\ $\mathrm{EH}=$ Encefalopatía hipóxica.
}


cerebral infantil (24.39\%) no presentan comorbilidad asociada, 84 pacientes con comorbilidad única $(29.26 \%)$, comorbilidad múltiple 133 casos (46.33\%). Conclusión: Las comorbilidades neuropsiquiátricas representan problemas médicos que significan igual o mayor repercusión en calidad de vida de los pacientes con parálisis cerebral infantil.

Palabras clave: Parálisis cerebral, comorbilidad neuropsiquiátrica, epilepsia sintomática.

Nivel de evidencia: III

\section{INTRODUCCIÓN}

La parálisis cerebral infantil (PCI), es consecuencia de una serie de alteraciones en el sistema nervioso central (SNC) que suceden en etapas biológicas del desarrollo (prenatal, neonatal o infancia). Se expresa por desórdenes que afectan movimiento y control postural corporal. ${ }^{1}$ Esta condición es estacionaria, sin embargo, hay condiciones comórbidas que pueden acompañar esta condición primaria, éstas tienen perfil heterogéneo y pueden comportarse en forma evolutiva. ${ }^{2}$

La PCI se clasifica con base en el tipo de compromiso motor en: espástica, extrapiramidal, atáxica, hipotónica y formas combinadas. En los pacientes con PCI espástica se describe el área corporal comprometida (mono, di, tri, para, o tetraparesia). ${ }^{3}$ La severidad del compromiso motor se califica de acuerdo al sistema de clasificación de la función motora gruesa (GMFCS, por sus siglas en inglés). ${ }^{4}$ La gran mayoría de los casos de PC se originan en etapa prenatal, aunque hay casos que derivan de factores neonatales o de la infancia. ${ }^{1}$

La prevalencia global es de 1.2-3.6/1,000 nacidos vivos, este valor se ha mantenido estable en las últimas tres décadas en diferentes países, independientemente de variables socioeconómicas. ${ }^{5,6}$ En relación con factores de riesgo específicos, la prematurez y el peso bajo al nacer son las dos condiciones con mayor riesgo relativo en la etiología de PCI. ${ }^{7}$

El tipo de comorbilidad en pacientes con PCI es heterogéneo y con frecuencia representa igual o mayor importancia en el manejo médico a largo plazo que la condición original que presentan estos pacientes. ${ }^{4,8-12}$

Comorbilidad se define como el trastorno o trastornos que acompañan a una enfermedad primaria. Implica la coexistencia de dos o más patologías médicas en un mismo paciente y pueden o no estar relacionadas en su fisiopatogenia. Se clasifica en tipo comorbidity, and multiple (two or more conditions in the same patient), was present in 113 cases (46.33\%). Conclusion: Neuropsychiatric comorbidities represent medical problems that mean equal or greater impact on the quality of life of patients with infantile cerebral palsy.

Keywords: Cerebral palsy, neuropsychiatric comorbidities, symptomatic epilepsy.

Level of evidence: III

I o comorbilidad primaria aquélla que es consecuencia directa de la condición biológica original, y tipo II o comorbilidad secundaria aquélla que es un desorden cuyo origen es independiente de la enfermedad original.

En la comorbilidad tipo I en pacientes con PCI encontramos: discapacidad cognitiva, trastornos de lenguaje, amaurosis, anacusia y epilepsia, así como diversas alteraciones neuropsiquiátricas.

También existe comorbilidad tipo I en otros órganos y sistemas fuera de sistema nervioso central. En este campo destacan las condiciones ortopédicas, gastroenterológicas, neumológicas y de nutrición. Condiciones que también, para obtener la mejor calidad de vida, deben ser evaluadas en forma integral.

En la comorbilidad tipo II se incluyen seis áreas de posible repercusión, conductual, emocional, social, habilidades académicas, habilidades escolares, y repercusiones financieras.

Objetivo: definir las características de las condiciones comórbidas en una población con PC en seguimiento regular.

Es este estudio longitudinal de cohorte se establecen diagnóstico y clasificación de la condición primaria (PCI) y la o las comorbilidades asociadas. Se analiza la comorbilidad neuropsiquiátrica (CNP) que presenta esta población de pacientes con PCI, así como la respuesta a los tratamientos indicados para cada condición.

\section{MATERIAL Y MÉTODOS}

En este estudio longitudinal de cohorte se establecieron diagnóstico y clasificación de la condición primaria (PCI) así como la o las comorbilidades neuropsiquiátricas (CNP).

Se evaluaron pacientes con diagnóstico de PCI en seguimiento regular a partir de enero de 2013 hasta diciembre de 2019. Cada paciente fue evaluado por el mismo investigador. 
La CNP se definió como la coexistencia de alteraciones neurológicas (epilepsia, discapacidad cognitiva, por ejemplo) o neuropsiquiátricas (trastorno por ansiedad, depresión, por ejemplo). No se incluyeron comorbilidades en otros órganos y sistemas (cardiopulmonar, gastrointestinal y ortopédica).

Se establecieron valores demográficos, diagnóstico primario, presencia y tipo de CNP, esquemas terapéuticos y respuesta al tratamiento.

\section{RESULTADOS}

Se evaluaron 287 pacientes con PCI, en seguimiento regular de enero de 2013 a diciembre de 2019. La distribución por sexo presenta ligero predominio de pacientes del sexo masculino (hombres $\mathrm{n}=158$, $55.05 \%$ /mujeres $\mathrm{n}=129,44.95 \%$ ).

La distribución por grupo de edad se presenta en la Figura 1, es interesante señalar que el rango de edad se expande desde un mes hasta los 57 años. La media de edad es nueve años ocho meses. Son menores de 12 años de nuestra serie el 65.16\% $(\mathrm{n}=187)$.

La etiología se presenta en la Figura 2, la encefalopatía hipóxica (EH) es la primera causa con 212 pacientes $(73.86 \%)$, interesantemente hay más casos en neonatos a término que en prematuros ( $\mathrm{n}$ $=117$ y $\mathrm{n}=95$, respectivamente). El $21.13 \%$ restante está integrado por seis grupos causales, llama la atención el registro de pacientes con disgenesia ( $\mathrm{n}=46,16.02 \%)$. En seis $(2.09 \%)$ no se identificó etiología.

La clasificación clínica de nuestros pacientes con PCI muestra predominio de pacientes con PCI espástica ( $\mathrm{n}=169,58.88 \%)$, la PCI hipotónica representa el segundo grupo más frecuente $(\mathrm{n}=58,20.02 \%)$. En proporción igual se presentan los pacientes con PCI

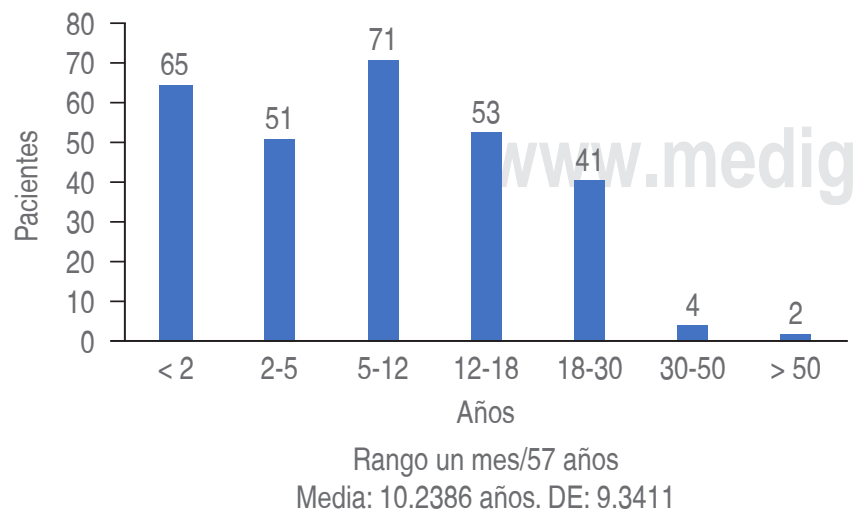

Figura 1: Distribución por grupos de edad.

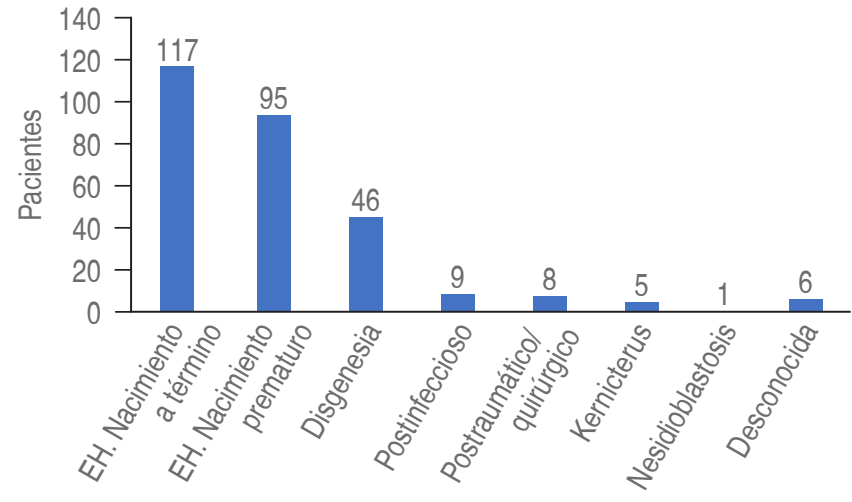

Figura 2: Etiología.

extrapiramidal y PCI combinada $(\mathrm{n}=30,10.45 \%$ para cada uno de estos dos grupos).

Con relación a la clasificación topográfica, encontramos que predominan los pacientes con PCI secundaria a compromiso hemisférico unilateral aquéllos que presentan monoparesia o hemiparesia $(\mathrm{n}=$ $173,60.27 \%)$. Fueron 70 pacientes con cuadriparesia (40.46\%), $24(13.87 \%)$ con paraparesia y $10(5.78)$ con diparesia $(5.78 \%)$.

La información anterior define las características clínicas de nuestros pacientes con PC.

Los resultados acerca de la condición de comorbilidad se presentan a continuación. Hay pacientes que cursan sin comorbilidad y otros pacientes presentan dos, tres o más condiciones comórbidas coincidentes a la PCI.

En esta serie, hay 70 pacientes con PCI (24.39\%) que no presentan comorbilidad asociada. Encontramos 84 pacientes con única comorbilidad asociada a PCI $(29.26 \%)$, La presencia de comorbilidad múltiple, que definimos como la presencia de dos o más diagnósticos en un paciente con PCI, se integra por 133 casos (46.33\%).

Son 113 pacientes que tienen dos o más condiciones comórbidas, por lo tanto, se presentarán los resultados con base en el número de diagnósticos y no de pacientes. El tipo y número de diagnósticos de CNP en esta serie se presentan en la Figura 3.

De los 287 pacientes con PC, 197 presentaron CNP, se integran 398 diagnósticos de comorbilidad, ya sea única o múltiple. La comorbilidad más frecuente es epilepsia ( $\mathrm{n}=136,34.17 \%)$, el segundo lugar es discapacidad cognitiva $(\mathrm{n}=97,25.12 \%)$. El resto de los grupos diagnósticos están descritos en la Figura 4.

Las alteraciones neuropsiquiátricas, en donde se incluyen trastorno por ansiedad, trastorno por déficit de atención e hiperactividad (TDAH), trastorno de con- 
ducta y depresión, representan una tercera parte del total de nuestros diagnósticos de CNP $(\mathrm{n}=129,32.41 \%)$.

Los esquemas de tratamientos farmacológicos se describen en la Figura 5, aquí nuevamente se relacionan con el número de pacientes. Una tercera parte (n $=91,33.79 \%$ ) están sin fármacos, ya sea porque la condición comórbida no tiene indicación de empleo de fármacos (discapacidad cognitiva, por ejemplo), o porque los objetivos del tratamiento se cumplieron y se resolvió tal comorbilidad. El mismo número de pacientes reciben tratamiento con fármaco único ( $\mathrm{n}=91,33.79 \%$ ). Pacientes con polimedicación, aquéllos que reciben tres o más medicamentos en forma simultánea, representan una sexta parte de esta serie ( $n=48,16.72 \%)$.

De los 196 pacientes en tratamiento farmacológico, se calificó el resultado de la farmacoterapia de la siguiente forma: óptima; cuando se alcanzó el mejor efecto en relación con la CNP en tratamiento (de esta forma será control anticonvulsivo total, o remisión de las alteraciones neuropsiquiátricas). Favorable; cuando se alcanzó una mejoría superior al 50\% de los objetivos del tratamiento. Mejoría leve; cuando el efecto terapéutico favorable fue menor al $50 \%$ del objetivo terapéutico. Nula; condiciones estacionarias. Deterioro; cuando el tratamiento generó incremento de las manifestaciones de la CNP, o la presencia de efectos colaterales adversos. Pérdida de seguimiento; al cumplirse un año sin contacto presencial o remoto con el paciente o su familia.

De esta forma, la respuesta óptima se alcanzó en 85 pacientes $(43.36 \%)$, favorable en $81(41.32 \%)$, leve mejoría en 15 (7.65\%), nula mejoría en seis (3.06\%), deterioro en tres $(1.53 \%)$ y pérdida de seguimiento en seis $(3.06 \%)$.

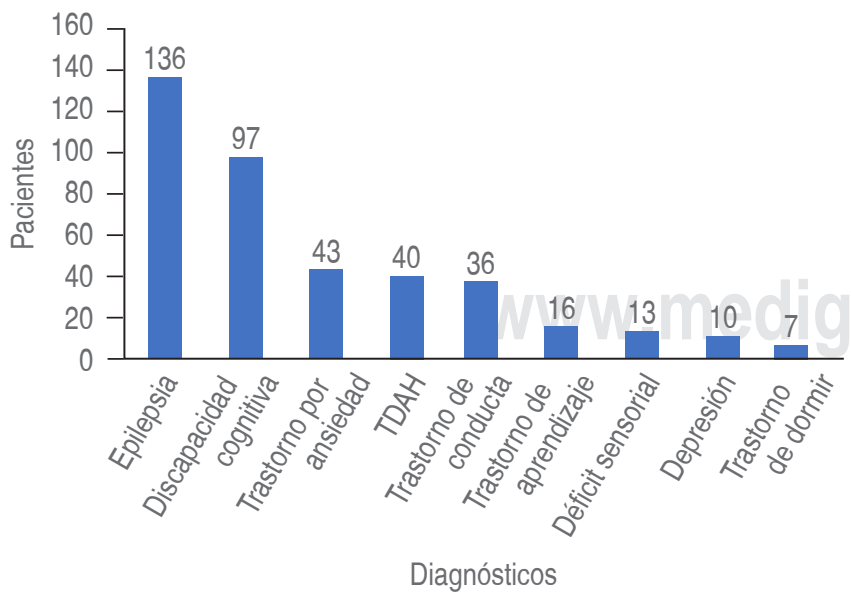

Figura 3: Diagnóstico de comorbilidad en pacientes con parálisis cerebral infantil.

TDAH = Trastorno de déficit de atención e hiperactividad.

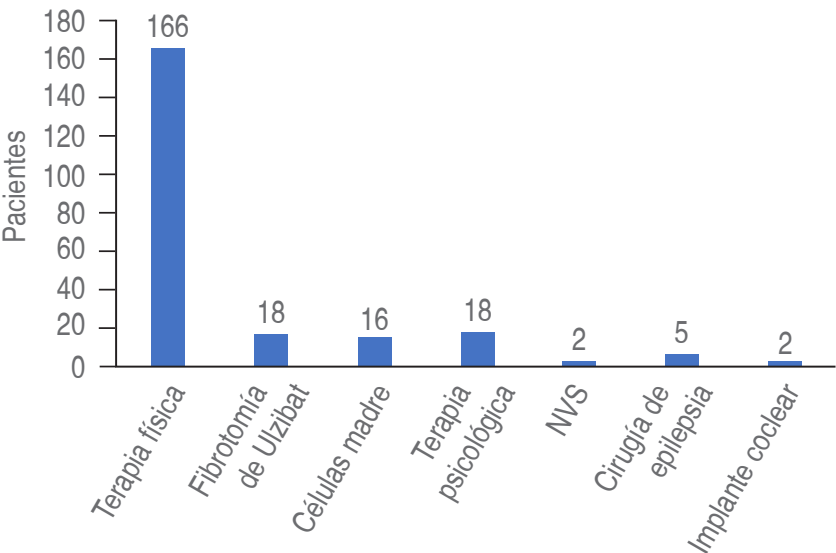

Figura 4: Comorbilidad en parálisis cerebral infantil en tratamiento no farmacológico.

NVS: estimulador de nervio vago (NVS por sus siglas en inglés).

En la Figura 6 se presentan los tipos de tratamiento no farmacológico. Aquí hay 227 tratamientos identificados de los 287 pacientes que componen esta serie. El recurso terapéutico más empleado es la terapia física. De los esquemas quirúrgicos, 18 pacientes fueron sometidos a fibrotomía de Ulzibat, cinco pacientes a cirugía de epilepsia, dos a estimulador de nervio vago (NVS por sus siglas en inglés) y dos a implante coclear.

\section{DISCUSIÓN}

En estos siete años de registro y seguimiento, la cohorte se integra con 287 pacientes con PCI.

Si bien la distribución por sexo presenta ligero predominio en pacientes del sexo masculino (hombres $\mathrm{n}=158,55.05 \% /$ mujeres $\mathrm{n}=129,44.95 \%$ ), es interesante señalar que en la población general de nuestro país predomina el sexo femenino (51.4\%). ${ }^{13}$

En esta serie prácticamente tres de cada cuatro pacientes presentan CNP primaria, el $46 \%$ de ellos tienen dos o más diagnósticos de CNP asociados a la condición primaria de PCI.

En el 70\% de nuestros pacientes, la CNP asociada (única o múltiple) puede explicarse por la etiología original; sin embargo, hasta en una tercera parte de nuestra población, la comorbilidad asociada se origina por diferente fisiopatogenia.

El análisis de las CNP lo realizamos con dos enfoques diferentes. Primero evaluamos la prevalencia en nuestra serie con la información nacional en población abierta, aquí se presenta la prevalencia de estos diagnósticos en nuestra serie con los resultados publicados en población mexicana por Caraveo y colaboradores. ${ }^{14-17}$ 
Al comparar la prevalencia de la CNP en nuestros pacientes y la prevalencia en población abierta de estos diagnósticos, encontramos una representación que va desde 2.84 hasta 33.84 veces mayor frecuencia, a excepción del diagnóstico de depresión el cual tiene una ligera mayor prevalencia en la población abierta que en nuestra serie. ${ }^{18,19}$

Analizamos la prevalencia de CNP con resultados reportados en la literatura internacional. Estos estudios fueron hechos con base en los registros de nacimientos y seguimiento por los sistemas nacionales de salud en los respectivos países. Esta comparación cuenta con una diferencia fundamental ya que nuestra serie es con base en la referencia de pacientes que presentan PCI, mientras que los otros estudios están hechos de acuerdo con registros poblacionales. ${ }^{13,14-16}$

En nuestra serie encontramos que los pacientes con PCI tienen mayor prevalencia de epilepsia que en todos los estudios internacionales comparados. ${ }^{18,19}$ En esta población, cuya CNP fue epilepsia, dividimos la edad de inicio en temprana cuando ésta se manifestó antes de los dos años de edad, y tardía cuando fue posterior a esa edad. En esta serie, 89 pacientes $(65.4 \%)$ tuvieron edad temprana de inicio, mientras que $48(34.6 \%)$ el inicio fue mayor a los dos años.

Diecisiete pacientes alcanzaron remisión epiléptica absoluta (control total de crisis y al menos dos años libres de medicamentos), 66 alcanzaron control total con monoterapia antiepiléptica, estos dos grupos, con el mejor resultado terapéutico, representan el $63.8 \%$ de nuestra serie. En el extremo opuesto, 47 pacientes $(36.1 \%)$ no alcanzaron control antiepiléptico, aun recibiendo tres o más fármacos.

El trastorno por ansiedad también tuvo una prevalencia mayor a la reportada internacionalmente.

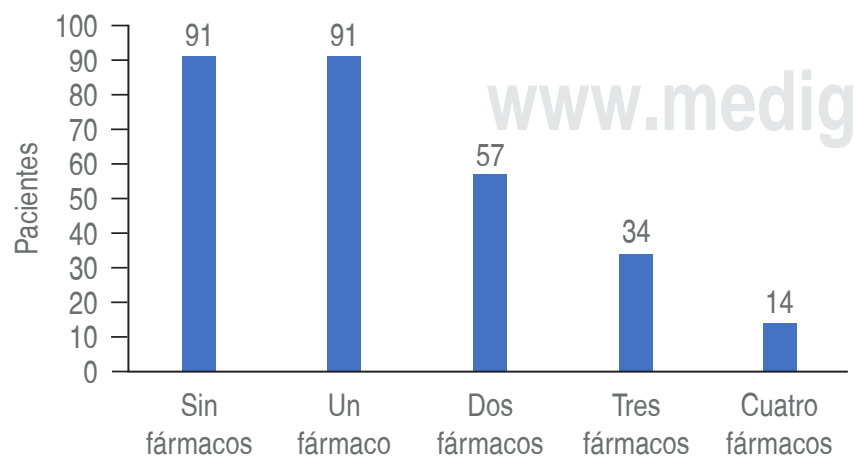

Figura 5: Comorbilidad y esquemas de tratamiento farmacológico.

\section{CONCLUSIONES}

Tres de cada cuatro pacientes con PCI presentaron CNP, de estos pacientes el $60 \%$ presentaron una o más condiciones asociadas. En dos terceras partes de nuestros pacientes con CNP la etiología corresponde a la condición primaria, mientras que en una tercera parte el origen de la CNP no puede explicarse por la causa de la PCI.

La CNP en pacientes con PCI representa una condición médica importante, es un factor extra que afecta la calidad de vida en estos pacientes, en muchas ocasiones repercute tanto o más que la repercusión en calidad de vida que la PCI en sí misma. Requiere identificarse y establecer terapéutica en forma individualizada. El panorama de respuesta al tratamiento es favorable, en nuestra serie obtuvimos una respuesta óptima o favorable en 186 pacientes (65.03\%).

En relación al pronóstico de la epilepsia en nuestros pacientes, dos terceras partes alcanzaron, ya sea remisión o control total manteniendo tratamiento en monoterapia.

Es indispensable evaluar y manejar a cada paciente con PCI en un esquema de atención multidisciplinaria. Las condiciones de CNP repercuten y agregan morbilidad a la condición médica de cada paciente.

\section{REFERENCIAS}

1. Bax M. Terminology and classification of cerebral palsy. Dev Med Child Neurol. 1964; 6: 295-297.

2. National Guideline Alliance (UK). Cerebral palsy in under 25s: assessment and management. London: National Institute for Health and Care Excellence (UK); (NICE Guideline, No 62), 2017.

3. Rosenbaum P, Paneth N, Leviton A et al. A report: the definition and classification of cerebral palsy. Dev Med Child Neurol. 2007; 49 (Suppl. 109): 8-14.

4. Shevell M, Dagenays L, Hall N. Comorbidities in cerebral palsy and their relationship to neurologic subtype and GMFCS level. Neurology. 2009; 72: 2090-2096.

5. Hollung SJ, Vik T, Wiik R, Bakken IJ, Anderson GL. Completeness and correctness of cerebral palsy diagnosis in two health registers: implications for estimating prevalence. Dev Med Child Neurol. 2017; 59: 402-406.

6. Galea C, Mcintyre S, Smithers-Sheedy H et al. Cerebral Palsy trends in Australia (1995-2009): a population-based observational study. Dev Med Child Neurol. 2019; 61: 186-193.

7. Novak I, Hines M, Goldsmith S, Barcley R. Clinical prognostic messages from a systematic review on cerebral palsy. Pediatrics. 2012; 130: e1285-312.

8. Brown JK, Eunson P, Bax M. Heterogeneity in cerebral pansy: variations in neurology, comorbidity and associated conditions. In: Bax M, Gillberg C, editors. Comorbidities in developmental disorders. London: Mac Keith Press, 2011, pp. 20-39.

9. Surveillance of Cerebral Palsy in Europe. Prevalence and characteristics of children with cerebral palsy in Europe. Dev Med Child Neurol. 2002; 44 (9): 633-640. 
10. Rosenbaum PL, Palisano RJ, Bartlett DJ, Galuppi BE, Russell DJ. Development of the gross motor function classification system for cerebral palsy. Dev Med Child Neurol. 2008; 50 (4): 249-253.

11. Delacy MJ, Reid SM; Australian Cerebral Palsy Register Group. Profile of associated impairments at age 5 years in Australia by cerebral palsy subtype and Gross Motor Function Classification System level for birth years 1996 to 2005. Dev Med Child Neurol. 2016; 58 (Suppl 2): 50-56.

12. Hafström M, Källén K, Serenius F et al. Cerebral palsy in extremely preterm infants. Pediatrics. 2018; 141 (1): e20171433.

13. Datos de INEGI. [Fecha de consulta 12 julio de 2020] Disponible en: https://inegi.org.mx.

14. Caraveo J, Medina-Mora ME, Rascón ML, Villatoro J, López EK, Juárez $\mathrm{F}$ et al. Características psicopatológicas de la población adulta urbana en México. Resultados de una Encuesta Nacional en Hogares. Instituto Mexicano de Psiquiatría, 1994, pp. 22-37.
15. Caraveo et al. Diagnóstico epidemiológico. [12 julio de 2020] Disponible en: http://www.salud.gob.mx/unidades/cdi/ documentos/pasm cap3.pdf.

16. Rodríguez J, Kohn R, Aguilar-Gaxiola S et al. Epidemiología de los trastornos mentales en América Latina y el Caribe. Washington DC: Organización Panamericana de la Salud, 2009, pp. 96-106.

17. Guía Clínica. Trastorno por Déficit de Atención e Hiperactividad. Hospital Psiquiátrico Infantil "Dr. Juan N. Navarro" 2009 ISBN 978-607-955.

18. Hollung SJ, Bakken IJ, Vik T et al. Comorbidities in cerebral palsy: a patient registry study. Dev Med Child Neurol. 2020; 62: 97-103.

19. Pruitt DW, Tsai T. Common medical comorbidities associated with cerebral palsy. Phys Med Rehabil Clin N Am. 2009; 20: 453-467. 